Itinéraires Itinéraires

Littérature, textes, cultures

2009-1 | 2009

Corpographèses

\title{
Singer ou de la répétition des mots à l'imitation des corps
}

Laurence Rosier

\section{(2) OpenEdition}

Journals

Édition électronique

URL : http://journals.openedition.org/itineraires/331

DOI : 10.4000/itineraires.331

ISSN : 2427-920X

Éditeur

Pléiade

\section{Édition imprimée}

Date de publication : 1 mai 2009

Pagination : 43-57

ISBN : 978-2-296-08444-5

ISSN : 2100-1340

\section{Référence électronique}

Laurence Rosier, "Singer ou de la répétition des mots à l'imitation des corps », Itinéraires [En ligne], 2009-1 | 2009, mis en ligne le 11 juin 2014, consulté le 01 juillet 2020. URL : http://

journals.openedition.org/itineraires/331; DOI : https://doi.org/10.4000/itineraires.331

Ce document a été généré automatiquement le 1 juillet 2020

\section{cc) (i) $\odot$}

Itinéraires est mis à disposition selon les termes de la licence Creative Commons Attribution - Pas d'Utilisation Commerciale - Pas de Modification 4.0 International. 


\title{
Singer ou de la répétition des mots à l'imitation des corps
}

\author{
Laurence Rosier
}

\author{
Hors des récits, les façons de marcher, de \\ s'asseoir, de parler et de rire, héler dans la rue, \\ les gestes pour manger, se saisir des objets, \\ transmettaient la mémoire passée de corps en \\ corps du fond des campagnes françaises et \\ européennes. \\ (Annie Ernaux, Les Années : 31)
}

\section{Introduction}

1 Nous partirons d'un cas précis qui a peu fait l'objet de commentaires (à l'exception notable des travaux de Bouvet 2001, Calbris 2003 cités par Chanay 2005, qui situe précisément son approche $\mathrm{du}$ discours et du langage dans une perspective pluridimensionnelle et plurisémiotique, de même Popelard et Wall 2005) : la place de la « répétition » des gestes, postures et attitudes que nous regrouperons, par commodité, sous la dénomination de corporalité, dans le champ des études consacrées au phénomène langagier du discours rapporté (désormais $\mathrm{DR}^{1}$ ) et sa mise en scène scripturale.

2 La codification sociale du corps et de ses représentations a peu intéressé l'analyse du discours : on se contente de l'affirmation : «le corps parle », métaphore classique mais qui n'a pas donné lieu à une véritable analyse discursive, l'analyse du discours laissant à l'anthropologie, à l'ethnologie ou encore à la philosophie la réflexion épistémologique et méthodologique des habitus corporels.

3 Par quels moyens linguistiques et discursifs le corps s'inscrit-il dans le fil du discours que l'on rapporte? Pourquoi est-ce que rapporter cette dimension corporelle est généralement déconsidéré du point de vue social ? Nous terminerons notre article par une proposition visant à spécialiser certains verbes servant à «reproduire "la 
corporalité, et par l'étude d'un verbe singulier, singer: ce lexème nous semble emblématique de la signification socioculturelle de l'acte de rapporter une corporalité, tel que l'illustre cet exemple récent trouvé dans la presse (il s'agit de l'arrivée en Angleterre de la première dame de France, Carla Bruni-Sarkozy), qui oppose précisément la citation d'un geste social codifié (la révérence ${ }^{2}$ ) à son affectation (singer) :

Lors de son arrivée dans la matinée à Windsor, chapeautée d'un bibi gris et vêtue d'une redingote ceinturée du même ton créée par John Galliano pour Dior, elle a fait une révérence impeccable [...]. La révérence, que le protocole, pourtant très strict, n'exigeait pas de l'épouse d'un chef d'état. Une génuflexion brève et naturelle. Un consentement aux codes de la monarchie britannique, qu'elle a "préféré » citer plutôt que singer. (Le Monde, 28 mars $2008: 3$ )

Le DR a été déplacé de ses limites grammaticales pour atteindre une approche plus large des phénomènes sociaux liés à la circulation des discours (comme la rumeur par exemple). On s'est interrogé sur la matérialité du discours que l'on rapporte (l'oral, l'écrit, les pensées, mais aussi les représentations, les opinions, les gestes), et sur l'activité même (qu'est-ce que " rapporter » : est-ce répéter, redire, reformuler ?). Mais peut-on répéter une corporalité ? Cette interrogation permet de pointer trois aspects relativement inédits du DR :

- Il s'agit de prendre rapporter au sens mimétique du terme : rapporter c'est alors imiter ${ }^{3}$, performance délicate dans la pratique quotidienne qui consiste à reproduire non seulement les discours des autres mais leur intonation, leur accent, leur geste, leur posture. Or, d'après les spécialistes de l'acquisition, l'imitation est une modalité permettant la mise en place d'interactions, elle n'est pas simplement un outil d'apprentissage mais un outil de communication (travaux de Nadel ou de Gaussier, voir le site de Ken Prepin: http:// ken.prepin.free.fr/). En 2005, la revue Terrain a par ailleurs publié un numéro consacré aux approches anthropologiques de l'imitation qui envisageait ses multiples aspects, à la fois comme « une stratégie parodique, une compétence cognitive, un moyen de transmission sociale, un principe de l'apprentissage social ou un mécanisme d'adaptation » (Dias 2005, en ligne), bref... un mécanisme extrêmement complexe qui dépasse la simple copie. Modestement, il s'agit ici de pointer un fait linguistique et d'envisager l'imitation comme une facette particulière des discours que l'on rapporte, tout en prenant en compte les dimensions socioculturelles des représentations de l'imitation telles qu'elles sont en circulation dans le discours social.

- Il s'agit d'envisager la théâtralité de l'acte même de rapporter, la mise en scène de l'autre à travers soi, qui possède une signification sociale spécifique.

- Il s'agit de pointer la nécessaire typification de la dimension corporelle de l'autre : sans aller jusqu'au travestissement (où l'on se fait passer pour un autre), on peut « contrefaire » son corps comme on contrefait sa voix, signalant qu'il y a non seulement un autre qui parle mais une autre corporalité qui est mise en scène selon des schémas corporels partagés par le sens commun et une communauté langagière spécifique.

\section{Ce que rapporter le geste et le corps signifie}

5 Nous considérons le discours rapporté au-delà des traditionnelles transpositions morpho-syntaxiques reprises dans les grammaires. Le discours rapporté est aussi et surtout pour nous une pratique sociale, à la signification ambivalente : rapporter, ce peut être dénoncer, faire acte de délation. Mais ce peut être aussi, au contraire, 
témoigner, au nom de la justice et de la fidélité des faits et propos rapportés, dans le cadre d'un procès par exemple. L'axiologie sociale de la pratique est donc double.

On retrouve cette dualité lorsqu'il s'agit de rapporter une dimension corporelle : est-il convenable, permis socialement, de s'approprier, de réénoncer le corps de l'autre? On peut aussi remonter en amont de cette question, en se demandant s'il est tout simplement possible de rapporter la corporalité d'autrui et ce que cela signifie. Bien entendu, on pense immédiatement à des situations fictionnelles, au cinéma ou à la télévision, où des acteurs endossent des personnages caractérisés par une voix, des gestes, des attitudes, une personnalité. Mais il n'y a pas, dans ce cadre-là, de corporalité rapportée au sens strict du terme puisqu'il y a identification entre l'acteur et son personnage et non dédoublement et exhibition d'une corporalité autre. Nous visons plutôt des situations où, à des moments ponctuels caractérisés, pour rapporter un propos d'autrui, non seulement nous empruntons les mots de l'autre, mais nous cherchons aussi à imiter la voix et le corps de l'autre dans leurs dimensions multiples (accent, intonation, timbre, geste, posture, allure). Les travaux cités en introduction parlent de «rôles endossés» et Chanay (op.cit.) ébauche une typologie des dédoublements énonciatifs et de leurs marqueurs "voco-posturo-gestuels » en usant des termes rejouer et mimer. La pratique existe, même si elle reste peu décrite.

7 Revenons donc à la signification sociale de l'acte même qui oblige à affiner ce que rapporter une corporalité veut dire. Il faut distinguer le fait de répéter simplement un geste (dans l'imitation immédiate ou différée dans le temps), de l'appropriation plus large d'une attitude corporelle, de ce que Bourdieu appelle un schéma corporel, signe distinctif d'un rapport de places et de classes dans une société valorisant les convenances et la retenue (la tenue étant, dit Bourdieu, « la manière légitime de tenir son corps et de le présenter ", $1979: 214$. Nous reviendrons plus avant sur ce point). La répétition d'un geste peut être attachée à la transmission patrimoniale d'un savoir-être ou d'un savoir-faire (les manières d'être poli ou les gestes d'un métier manuel par exemple) : on répète les gestes de son père, de sa mère ${ }^{4}$, d'un professeur, d'un artisan. Il s'agit alors moins d'une répétition mimétique que d'une incarnation ou d'une appropriation de gestes patrimoniaux ou techniques. Nous laisserons de côté ces dimensions plus spécifiquement anthropologique et ethnologique du geste pour nous focaliser sur la dimension sociale normative à laquelle elles s'articulent. La transmission de ces gestes relève tout autant de l'identité corporelle propre que de la transmission de pratiques socioculturelles et de représentations typiques. Cette dimension de perpétuation de postures et de gestes ancestraux a une image sociale positive.

8 Il existe une autre manière de répéter un geste, en le citant, ce qui en fait un geste de mémoire, avec des valeurs plus ou moins négatives. Dans le cadre spécifique de l'insulte (terrain où la dimension corporelle est intimement liée à des pratiques transgressives et à une mise en avant de la dimension organique du corps - qui est généralement bannie des convenances -, ce que révèlent des expressions comme cracher ou vomir des insultes), parmi les gestes considérés comme insultants, on trouve cette dimension citationnelle du geste. Son interprétation donne lieu à des discussions juridiques et à des décisions judiciaires diverses en vertu précisément de cet effet citation: lorsque l'attaquant de la Lazio de Rome, Paolo di Canio, fait le salut fasciste lors d'un match contre Livourne en janvier 2005, il prétend avoir fait un « salut romain » et ne s'estime donc pas coupable d'un comportement insultant. L'argument vise à dire qu'on 
reconnaît bien qu'il y a citation mais d'un geste autre, c'est-à-dire ancré dans une autre dimension historique (c'est un procédé de révision historique bien connu des spécialistes). La dé-mémoire (Robin citée par Paveau 2006 : 110) du geste fait primer un contexte qui vise à annuler la signification historique et donc la dimension citationnelle spécifique. Ainsi, en 1999, le tribunal correctionnel de la ville d'Anvers en Belgique acquitte un conseiller provincial du Vlaams Blok (parti d'extrême droite flamand) accusé d'avoir exécuté un salut hitlérien lors d'une séance du conseil provincial car le tribunal n'a pas considéré que le geste effectué avec le bras constituait réellement un salut hitlérien mais plutôt un «mépris grossier à l'égard d'un organe politique établi ». Par contre, le 4 février 2002, un élu du Vlaams Blok, encore, a fait un salut hitlérien lors de sa prestation de serments devant le nouveau conseil communal et a été condamné, témoignages écrits et photos établissant clairement l'identification du geste « lié au régime fasciste ». Citer un geste ouvre dès lors des perspectives pour l'étude de la transmission d'une corporalité sociale marquée par l'histoire et les tensions entre mémoire et dé-mémoire du geste et du corps. C'est moins la répétition, c'est-à-dire l'acte même qui est condamné ici que l'appel historique suscité par le geste cité.

9 Prise dans sa dimension strictement imitative, la répétition est considérée de façon généralement négative. La dimension mimétique - ce terme signifiant certes l'imitation mais aussi la représentation, voire la fiction et le mensonge - est, dans la tradition platonicienne, condamnée. Dans les manifestations formelles de la mimésis platonicienne, on trouve le discours direct comme preuve suffisante de mimésis, donc une forme de discours rapporté et, comme genre, le théâtre précisément dans sa dimension corporelle (Platon donne comme exemple imiter le bruit du tonnerre avec sa voix ou, avec son corps, un animal). Rapporter au sens d'imiter contrevenait à la conception esthétique de la beauté chez le philosophe antique.

C'est du point de vue des convenances sociales que nous appréhenderons l'imitation du corps de l'autre. En effet, pour nous, cette pratique est marquée. Pourquoi? Si on admettra qu'un humoriste-imitateur contrefasse la voix d'une personne célèbre ou qu'un enfant imite son grand-père en prenant une voix chevrotante et une pose de vieillard (ce qui suppose, nous y reviendrons, une série de représentations stéréotypiques des schémas corporels), on imaginera plus difficilement un journaliste relatant des propos échangés avec une personnalité médiatique en lui empruntant ses gestes et ses postures.

11 Dans une société, les prescriptions normatives régissent les façons de parler orales et, plus largement, gestuelles et comportementales, comme l'illustre cet extrait d'un guide des bonnes manières :

Si vous avez un accent terrien ou provincial prononcé, essayez de le perdre. [...] Prenez garde aux liaisons, certaines sont dangereuses. [...] Des locutions vicieuses sont pires qu'un mot cru ou grossier (Guide Marabout 1951 : 72-73).

Gardez-vous d'un ton de voix trop haut. Et, du point de vue des gestes, des gros mots et des gestes superflus (idem : 157).

12 En effet, les guides et manuels de savoir-vivre se livrent à une apologie de la neutralité corporelle et vocale (voir Paveau et Rosier 2008), poursuivant dans ses derniers recoins l'excès, le « trop ». Dans son dernier roman paru en 2008, Annie Ernaux le rappelle très justement, en décrivant cette norme socioculturelle qui pesait essentiellement sur le comportement des filles : 
La honte ne cessait de menacer les filles. Leur façon de s'habiller et de se maquiller, toujours guettée par le trop : court, long, décolleté, étroit, voyant, etc., la hauteur de leurs talons, leurs fréquentations, leurs sorties, leurs rentrées à la maison, le fond de leur culotte chaque mois, tout d'elles étaient l'objet d'une surveillance généralisée. (Les Années : 73) sur le savoir-faire et le savoir-être en société qui concerne bien entendu la corporalité. Qu'en est-il de la notion d'imitation dans ce cadre? Quelles en sont les règles sociales implicites et explicites?

\section{Corps et convenances : la condamnation sociale de l'affectation}

La place qu'occupe le corps dans le savoir-vivre est importante parce qu'il s'agit essentiellement de signifier une position sociale. «La distribution entre les classes des propriétés corporelles » (Bourdieu, $1979: 210)$ a produit une sémiologie du corps, basée sur un imaginaire codifié et stéréotypé, qu'il soit de type sexiste (une femme doit être discrète), ethnoculturel (l'Italien parle avec les mains) ou social (le baisemain se fait sans que la bouche touche la main), mais cette socialité est articulée à un impératif naturaliste : il s'agit surtout de ne pas montrer qu'on fait des efforts pour acquérir les convenances corporelles admises. Or, «les stéréotypes de la convenance sont, par la présentation du corps, une manipulation de la distance sociale » (Giard et Mayol 1980: 32) et les manuels de savoir-vivre l'ont parfaitement compris : ils insistent sur une série de pratiques de politesse et de convenances sociales liées à des rituels sociaux (mariage, repas, enterrement, vacances, etc.). Il s'agit de gestes liés, ou non, à des usages d'objets (par exemple prendre tel couvert à tel moment du repas) et d'attitudes générales liées à l'identité (comment être élégante) et aux relations sociales (qui saluer en premier lieu dans une soirée). Acquise, cette corporalité devient naturelle aux yeux des autres et n'est donc pas perçue comme l'emprunt d'une corporalité d'autrui. C'est la tradition du naturel comme seconde nature.

Quant à la notion d'imitation, elle est ambiguë: la fonction d'apprentissage de l'imitation est reconnue dans le domaine des spécialistes du développement de l'enfant (Piaget) où l'imitation est la première manifestation de la fonction symbolique de la représentation.

Dans le registre de la codification sociale, les manuels de savoir-vivre, d'hier et d'aujourd'hui, qu'ils soient de politesse, de convenance ou de bonnes manières, participent de la «civilisation des mœurs» (selon les termes du philosophe Norbert Elias) et vantent la grande force de l'exemple dans l'éducation des enfants. L'imitation y est vue comme la façon la plus naturelle d'acquérir les bonnes manières et elle devient constitutive d'un éthos social :

La plus grande force des parents, à cette heure surtout où leur rôle va progressivement consister moins à commander qu'à guider, réside surtout dans le bon exemple qu'ils donnent et dans l'affection et l'estime qu'ils savent inspirer. Le jeune enfant, naturellement imitateur, s'essaie à faire ce que font les parents [...] un père qui veut avoir un fils de valeur ne peut rien faire de mieux pour $\mathrm{y}$ arriver que de commencer par être lui-même un père que l'on peut imiter. (Savoir-Vivre ${ }^{5}$ : 78) 
17 Les manuels de savoir-vivre proposent donc une police du corps, où chaque geste appris est mesuré et sert à composer une attitude sociale :

Tous les gestes de la vie servent à composer notre personnage. Vous pouvez fumer vingt-quatre ou quarante-huit cigarettes dans une journée et laisser passer votre vice presque inaperçu. Avec cinq ou six cigarettes par jour vous pouvez être odieux à votre entourage. Gardez la mesure et l'élégance dans chacune de vos actions (Guide Marabout 1951 : 83).

18 Mais l'imitation sans vertu éducative est proscrite et sa condamnation révèle les corporalités stéréotypiques socioculturelles (dans nos exemples la vamp, le canard, la pauvresse traîne-savate, Chariot et sa composante humoristique) et leur dimension démonstrative :

Madame, évitez les grandes enjambées si peu féminines et freinez vos bras s'ils ont tendance à imiter le balancier d'une horloge ; n'ondulez pas de la croupe, n'imitez pas les mannequins des grandes maisons en jouant les vamps; ne vous traînez pas non plus à la manière d'une pauvresse qui manquerait de force pour lever les pieds. Évitez d'imiter les canards qui claudiquent les pattes en dedans, mais ne tombez pas dans l'excès contraire en imitant Chariot. (Guide Marabout, 1951 : 153)

19 Ainsi rapporter une corporalité autre, c'est-à-dire la mettre en scène de façon ostentatoire, enfreint les normes sociales à plus d'un égard. Rapporter une corporalité est nécessairement réducteur donc typifiant et stigmatisant : cette action contrevient à l'idée de mesure et de "normalité » corporelle, elle relève moins du geste du corps que de la gesticulation, elle sur-marque aussi des différences sociales, ethniques, sexuelles.

Sous couvert d'une certaine uniformisation sociale, les manuels de savoir-vivre confortent les différences de classe et de sexe : ainsi les manuels nous diront quels sont nos devoirs «envers les supérieurs, ses égaux, les inférieurs " (Savoir vivre 1946, présentation du livre $1: 13)$.

21 C'est donc la dimension sociale du fait de « citer » le corps de l'autre, allant jusqu'à une appropriation généralement considérée comme stigmatisante (on est proche de l'insulte) et surtout usurpée, qui est dévaluée. Dans le Dictionnaire raisonné de la politesse et du savoir vivre (1995) dirigé par Alain Montandon, à l'article «affectation», nous lisons sous la plume de Mercedes Blanco :

Le parvenu est celui qui, s'étant glissé dans la classe dominante, ne peut qu'y singer ses façons et ses valeurs. Il affiche une sorte de caricature de distinction, une mauvaise imitation. (1995: 19)

Sortir de la classe à laquelle on était socialement assigné ne peut, paradoxalement, se montrer : l'affectation, c'est l'excès ou l'effort, c'est le trop que nous avons évoqué cidessus. C'est à cette occasion que le lexème singer nous est apparu comme particulièrement emblématique d'une activité sociodiscursive particulière qui actualise exclusivement le sème négatif du verbe imiter. Comme on oppose le verbe dire, plutôt neutre, au verbe prétendre, qui jette la suspicion sur le degré de vérité du discours que l'on cite, nous proposons une triade verbale qui comprend les différentes modalisations de l'acte de rapporter une corporalité et le jugement social qui en découle : citer-imitersinger. 


\section{De citer à singer : des reprises de la corporalité d'autrui}

23 L'imitation, nous l'avons dit, est l'une des conduites possibles pour acquérir des habitus de classe et des hexis corporelles. Elle véhicule également une représentation commune, mais qui fascine aussi scientifiques et écrivains (Dias 2005 : 5) : la faculté d'imitation attribuée au singe. Dans nos perceptions ordinaires, les comparaisons avec le monde animalier dans les expressions répéter comme un perroquet ou singer un comportement illustrent une dimension foncièrement négative.

En travaillant davantage sur la réénonciation et la recontextualisation des discours, nous nous sommes attachée à la dimension mimétique qui sous-tend l'acte de rapporter: les verbes comme imiter ou singer comportent bien une dimension non seulement orale mais aussi gestuelle et corporelle, en plus d'une signification sociale. Singer c'est vouloir être autre sans y parvenir, c'est manquer le naturel, c'est s'approprier des codes, acquérir des habitus non transmis naturellement par l'éducation et le milieu culturel. Dès lors, rapporter représente une pratique moins d'appropriation de l'autre que d'effacement de soi au profit d'un comportement, verbal ou non, reconnu comme valorisé socialement mais qu'il s'agit de rendre imperceptible.

Dans le cadre linguistique lato sensu, rappelons encore qu'on peut rapporter, sous différentes modalités (volonté, interdiction...), des discours écrits, des paroles et, plus généralement, des interactions : dans ce cas, on doit alors s'interroger sur les modalités de reprise du paraverbal et du non-verbal comme les intonations, les gestes, les postures qui relèvent de la représentation quasi théâtrale non seulement du discours de l'autre mais de sa corporalité. On peut rapporter des pensées (se dire, penser, croire), des émotions (s'étonner, craindre), des perceptions (entendre, voir, sentir), des actes (à l'aide de verbes performatifs comme promettre, condamner), mais aussi des gestes et même une "certaine allure » : ne dit-on pas couramment que l'allure d'un tel rappelle l'allure d'un autre, effet de mimétisme fortement exploité récemment dans les médias sur les ressemblances entre le couple Kennedy et le couple Sarkozy (le précédent: Nicolas et Cécilia)? Les prescriptions en matière de mode ne font pas autre chose : copier la dégaine, avoir l'allure de, l'élégance de, être sexy comme Kate Moss (accroche du magazine Vogue en avril 2008), le désir de conformité sociale passe par une imitation où le rapport au modèle est complexe. Il s'agit d'évoquer sans apparaître comme la copie d'un original. Par ailleurs, il existe toute une série de verbes qui montrent comment l'acte de rapporter a des intentions différentes suivant la forme du discours qu'il suppose : caricaturer, parodier, pasticher, feindre sont des verbes qui dénotent une attitude négative à l'égard de celui dont on rapporte le discours ou le corps comme pour celui, d'ailleurs, qui emprunte ces formes pour rapporter.

Enfin, l'imitation recèle une capacité comique reconnue par nos sociétés: l'effet recherché est pittoresque, théâtral et les humoristes ne s'en privent pas. Cependant la mise en représentation de la voix et du corps de l'autre est une stigmatisation par simplification selon des stéréotypes. À l'écrit, cette typification passe parfois par des transcriptions phonétiques (plus ou moins approximatives et stéréotypiques) pour tenter de rendre la prononciation particulière d'un personnage romanesque, même s'il est reconnu que les guillemets ne sont pas là pour nous indiquer qu'on doit de façon mimétique reproduire l'intonation du discours original. «Tout au plus le lecteur se guidera-t-il sur eux pour modifier le ton de sa voix et imiter, dans les limites du convenable, celles des personnages " (Weinrich 1973: 206). À l'oral, l'imitation de 
l'accent ou d'une caractéristique vocale propre à une personne n'est pas une activité banale, tout comme, et c'est encore plus marqué, l'imitation de la corporalité. Imiter un accent s'appuie aussi sur des représentations stéréotypées, par exemple dire putaing ou peuchère pour imiter l'accent de Marseille ou remplacer « $b$ » par " $p$ » pour imiter l'accent allemand. Or les accents sont stigmatisés socialement, comme toute conduite du corps "déplacée ", inconvenante, excessive. Les caractéristiques de la grossièreté laissent d'ailleurs une large place à une description de la mauvaise conduite du langage et du corps : le laisser-aller, le sans-gêne, le manque de tact, l'indiscrétion « de ceux qui parlent et rient fort ou font de grands gestes» (Dominique Bertrand dans Montandon 1995 : 453). L'enflure verbale et gestuelle est donc très strictement balisée socialement: elle peut être utilisée à des fins comiques, dans des cadres où la théâtralité du corps est permise - toujours à des fins humoristiques, par exemple la capacité de raconter des blagues et d'imiter des accents et des postures.

L'action de rapporter une corporalité couplée à celle de rapporter un discours, en le montrant, fait partie d'un dialogisme généralisé où affleurent (on pourrait dire s' actualisent), selon un coefficient linguistique plus ou moins marqué, non seulement le dire ou le pensé d'autrui mais sa dimension corporelle typifiée et rapportée à des comportements sociaux plus ou moins codifiés. Ce qui ne veut pas dire qu'on ne puisse rendre l'autre dans son individualité, par exemple rapporter la parole d'une personne et imiter un tic, comme celui de hausser constamment les épaules en parlant: ce comportement n'est pas commun au sens social, il relèverait donc plutôt d'une «idiocorpographie » que d'une «sociocorpographie »: mais l'alliance d'un discours et d'un geste pour typer un individu participe d'une activité sociale de mise en scène de l'autre. L'intention communicative et la nécessité d'identifier cette corporalité rapportée obligent à emprunter des codifications acceptées dans une société donnée (par exemple, je veux rapporter une conversation avec mon ami Marius qui habite le Sud : j'emprunte ce qui est communément perçu comme un accent du Sud, chantant, etc., et je vais faire de grands gestes, reproduisant donc là l'ethnotype classique du méridional fanfaron tel qu'il a été étudié par Robert Lafont [1977]).

La triade de verbes évoquée ci-dessus (citer-imiter-singer) illustre une échelle axiologique des corporalités rapportées, allant de la signification sociale positive de la reproduction de la corporalité de l'autre, à la signification sociale négative qui relève de deux sous-axes : soit c'est la performance elle-même qui est ratée et c'est le mimeur qui est dévalorisé, soit la performance est réussie et c'est le mimé qui en prend pour son grade.

Prenons le verbe citer de notre premier exemple : citer s'applique à un geste social, qu'il relève de conventions partagées (par exemple faire une révérence, saluer) ou qu'il soit un geste qui ait acquis une notoriété historique (comme dans notre exemple du salut fasciste public) ou plus éphémère (par exemple dans les cours d'école, répéter le geste du héros du film éponyme Brice de Nice lorsqu'il «casse ses adversaires » et qu'il produit un mouvement de la main allant de haut en bas et de la droite vers la gauche). Citer, c'est re-faire en montrant qu'il s'agit du faire d'un autre (individu ou groupe social).

Prenons ensuite le verbe imiter, circonscrit aux emplois où il est utilisé pour rapporter les gestes, postures, attitudes : l'imitation de la corporalité d'autrui se caractériserait par une intention spécifique avec une visée communicative particulière. Traitée du point de vue de la performance, l'imitation d'un autre, qu'on doit aussi reconnaître 
comme autre pour qu'on puisse évaluer ladite performance, est une compétence qu'on peut juger positivement mais liée à des pratiques sociales très spécifiques où la théâtralité est permise et où la stigmatisation de la corporalité fait partie du genre. Par exemple, de nombreux humoristes imitent des personnalités ou mettent en scène des types sociaux avec leurs tics de langage, leurs manières de geste et de corps. Ce qui permet d'appréhender ce genre d'imitation comme relevant du discours rapporté, c'est le décalage énonciatif produit entre le producteur physique de la parole (l'humoriste) et les personnages qu'il endosse et joue. Personnages médiatiques (par exemple, dans une gamme classique, Canteloup, qui débuta dans les célèbres Guignols de Canal +, fait l'humoriste-animateur dans l'émission hebdomadaire de Michel Drucker le dimanche après-midi et propose toute une palette d'imitations de la voix et de la corporalité du monde politique et artistique) ou types sociaux (les personnages populaires des Deschiens ou des petites annonces d'Élie Semoun), les imités se voient donc rapportés dans leur dimension voco-posturo-gestuelle. L'imitation à la perfection, expression courante, implique cependant toujours un repérage de deux voix, celle de l'imitateur et celle de l'imité, individu ou type.

Prenons enfin le verbe singer, qui a incorporé une dimension sociale spécifique négative et examinons quelques exemples tirés de la littérature, qui est un bon corpus pour révéler les représentations sociales partagées. Singer est le verbe qui a incorporé une dimension de distinction sociale affectée, comme l'attestent les deux exemples qui suivent, l'un du XVIII ${ }^{\mathrm{e}}$ l'autre du début du $\mathrm{Xx}^{\mathrm{e}}$ siècle :

(1) Mais mon serrurier et le laquais de monsieur, reprit la marquise, n'étant pas dans ce cas, pourquoi ces animaux-là jouent-ils ? Pour singer les grands, madame, répliqua Brochure, et se donner un air. Vous savez qu'à Paris rien n'est si arrogant que le petit peuple et la valetaille. (Chevrier, Le Colporteur : histoire morale et critique, $1761: 149)$

(2) Aussitôt parvenus à la fortune... vous n'avez plus qu'une idée : nous singer... C'est nos hôtels, nos terres, nos manies, nos vices qu'il vous faut... nos vieux noms glorieux... et jusqu'à nos vieux meubles. (Octave Mirbeau, Les affaires sont les affaires, $1903: 131)$

Singer implique une théâtralisation jugée de façon négative pour le singeur: celui-ci semble usurper une place sociale et le verbe s'applique davantage à ce déplacement social où le singeur est repéré par « les indices subtils des manières ou du maintien où se trahit l'effet de conditions d'existence différentes des conditions présentes" (Bourdieu 1979 : 122). La volonté de mobilité sociale - l'ascension sociale - se superpose à un souci de conformité aux normes perçues comme marquant l'appartenance à une classe ou à un groupe. Ce désir de conformité est précisément l'objet d'opprobre, comme le montrent les deux exemples précédents, où des termes stigmatisants (animaux, parvenus) indiquent à la fois la dimension mécanique de l'imitation «sans génie » et sa fonction d'ascenseur social (le besoin de parvenir).

Les caractéristiques de cette imitation ratée relèvent d'une attitude sociale générale sentie comme transgressive (selon le principe "on reste à sa place») et le singeur apparaît comme un usurpateur de classe. En ce qui concerne plus précisément son usage en discours, on voit que le verbe singer permet d'introduire un discours direct, forme qui repose sur l'idée commune de littéralité et de fidélité dans le rendu du discours d'autrui, alliant les paroles, les gestes, l'intonation. L'exemple d'André Dhôtel ne précise pas ce qui dans singer relève du verbal ou du paraverbal. Seule l'intention moqueuse est perceptible et le corps est laissé dans l'ombre : 
(3) Paul se mit à singer $\mathrm{M}^{\text {me }}$ Terpoint :

- Et ça prétend connaître la vie ! Quand on vous dit qu'il y a des choses qu'on voit sur les journaux et qui existent. Puis il s'assit dans l'herbe et soupira.

(André Dhôtel, Le Ciel du faubourg, 1956 : 184)

sociaux : lorsqu'on dit singer la douleur, on visualise l'image de quelau'un se roulant par terre, hurlant l'interjection correspondante ("aïe ») sans l'éprouver réellement. Dès lors, des émotions ayant leur expression stéréotypée seront plus facilement singées : singer la peur, la joie, ou l'effroi comme dans l'exemple qui suit.

(6) [...] me voici arrivée à l'endroit où il me faut singer l'effroi, j'arrondis mes lèvres,

j'ouvre mes yeux tout grands, ma voix monte, vibre...

"Quand on a peur du loup, du vent, de la tempête... "

(Nathalie Sarraute, Enfance, $1983: 62$ )

C'est sur ces mêmes représentations sociocorpographiques que le singeur reproduit la corporalité du singé : celui-ci doit présenter des caractéristiques vocales, paraverbales ou plus largement physiques qui soient stigmatisables comme trait identitaire et social. L'acte de singer est alors défavorable au singé puisque sa corporalité est répétable, c'està-dire qu'il offre des traits marqués, prêtant le flanc à leur caricature. Dans notre exemple 7, ce sont l'éthos social du paysan, ses mauvaises manières et l'allure convenue d'une personne ivre :

(7) Les clients regrettent Jeanne. Ils singent la mère Coup-De-Tampon et ses manières paysannes, sa démarche le jour où elle $\mathrm{a}$ bu un verre de trop. Elle se venge en sabotant son service. (Eugène Dabit, L'Hôtel du Nord, 1929 : 225)

Cependant, lorsque c'est la dimension stigmatisante de la répétition corporelle qui prime et donc la mise en spectacle, à des fins burlesques, du corps d'autrui, le singeur réussit une performance et ce n'est plus lui qui pâtit alors de la sanction sociale, mais celui dont on imite, avec outrance, les manières et le corps. On rejoint là, d'une certaine manière, la tradition carnavalesque du bouffon dont la place sociale est précisément d'exhiber, pour en canaliser la subversion, le trop des discours et des corps.

Bien entendu, les emplois en contexte jouent avec les sèmes communs aux trois verbes et imiter et singer sont parfois utilisés l'un pour l'autre. C'est alors le cotexte et/ou le 
contexte qui permettent de déterminer si imiter suffit pour décrire l'action ou si singer s'impose. Mais il nous a semblé pertinent, si nous voulons distinguer des degrés de rapportabilité ou de citabilité du corps attelés à des significations sociales, de chercher à spécialiser, du point de vue théorique, ces verbes qui correspondent à des pratiques sociales de répétition et d'appropriation du corps différentes.

\section{Conclusions provisoires}

Depuis Saussure, on a paru oublier, en linguistique, que cette discipline était une sémiologie parmi d'autres, et que la langue fonctionnait dans le social avec l'ensemble des autres appareils sémiologiques que sont par exemple les images, les gestes et les corps. Interroger la place du corps dans les mécanismes de reproduction et de circulation des discours semble une piste susceptible d'apporter des éléments neufs dans l'appréhension de la dimension citationnelle : citer l'autre ne se réduit pas à une dimension purement linguistique, voire discursive. On cite les paroles d'autrui mais aussi son corps et cela témoigne d'un degré d'investissement du sujet dans son discours, sujet qui met en jeu son éthos social selon le degré de "réussite discursive " (Chanay, $2005: 245$ ) de sa capacité de mimeur, qu'il cite, imite ou singe.

\section{BIBLIOGRAPHIE}

Bourdieu, P., 1979, La Distinction. Critique sociale du jugement, Paris, Minuit.

Chanay, H. de, 2005, « Associations et dissociations énonciatives entre geste et parole :

polyphonie et dialogisme dans une interview de Jean-Claude Van Damme ", dans Bres, J. et al., Dialogisme et Polyphonie : approches linguistiques, actes du colloque de Cerisy, 3-9 septembre 2004, Bruxelles, De Boeck, p. 231-246.

Corbin, A., Courtine, J.-J. et Vigarello, G., 2006, Histoire du corps, Paris, Le Seuil, t. 3.

Dias, N., 2005, «Imitation et anthropologie », Terrain, $\mathrm{n}^{\circ}$ 44, p. 5-18.

Giard, L. et Mayol, P., 1980, L’Invention du quotidien : 2/ Habiter, cuisiner, Paris, Union générale d'éditions.

Guide Marabout du savoir-vivre de tous les jours, 1951, de G. Assailly et J. Baudry, Verviers, Marabout.

Lafont, R., 1977, « D'un autre luoc : la trilogia de Pagnol », Annales de l'Institut d'Études occitanes, nouvelle série, $\mathrm{n}^{\circ} 1$, p. 53-78.

Laloyaux, M.-M., 1946, Savoir-Vivre, Charleroi et Paris, Dupuis et fils.

Montandon, A. (dir.), 1995, Dictionnaire raisonné de la politesse et du savoir-vivre du Moyen Âge à nos jours, Paris, Le Seuil (articles « affectation » de M. Blanco, « corps » de D. Picard, « distinction » de M.-C. Natta, « grossier » de D. Bertrand).

Paveau, M.-A., 2006, Les Prédiscours. Sens, mémoire, cognition, Paris, Presses Sorbonne Nouvelle.

Paveau, M.-A. et Rosier, L., 2008, La Langue française. Passions et polémiques, Paris, Vuibert. 
Popelard, M.-D. et Wall, A. (dir.), 2005, Citer l'autre, Paris, Presses Sorbonne Nouvelle.

Rosier, L., 2008, Le Discours rapporté en français, Paris, Ophrys.

Weinrich, H., 1973, Le Temps, Paris, Le Seuil.

\section{NOTES}

1. Le discours "rapporté » ou "représenté » recouvre des formes marquées du dialogisme (discours direct, discours indirect, discours indirect libre) et des modalisations diverses qui attribuent le discours d'autrui (selon X).

2. Le Guide des convenances de Liselotte (édition de 1931) signale : "C'est un geste ancien qui est revenu à la mode. La révérence se réduit généralement à une simple flexion du genou. Elle est pratiquée presque exclusivement par les fillettes ou les jeunes filles saluant une dame. Les jeunes femmes peuvent la faire à une vieille dame. » (242)

3. Nous ne limitons pas le terme imiter au sens qu'il possède dans les études actuelles menées en psychologie du développement ou en neurocybernétique, mais nous tenons à signaler que c'est dans ce cadre qu'aujourd'hui on interroge la notion d'imitation, sa définition, son acquisition et l'évaluation des capacités imitatives, liées notamment à l'environnement et aux rôles des objets.

4. Dans L'Invention du quotidien, t. 2, Giard et Mayol parlent de la transmission des gestes de la cuisine et évoquent l'enfant qui « apprenait du regard l'activité culinaire auprès de sa mère ou de sa grand-mère » (223).

5. Cet ouvrage publié en Belgique en 1946 s'appuie sur les guides les plus connus comme Le Guide des convenances de Liselotte et Les Règles du savoir-vivre de la Baronne Staffe.

\section{RÉSUMÉS}

Cet article aborde les façons dont les gestes d'autrui, ses postures et ses attitudes (englobées dans le concept de matérialité) aussi bien que ses paroles, peuvent être rapportés, ainsi que la place de la répétition corporelle dans le discours indirect comme phénomène spécifique du langage. Par le biais d'un examen des valeurs sémantiques de trois verbes (citer, imiter, singer), il soutient que la citation du corps d'une autre personne indique un certain degré d'investissement du sujet dans son propre discours, sujet qui joue son ethos social sur le degré de réussite discursive de sa performance.

This article addresses the ways in which the other's gestures, postures, and attitudes (subsumed by the concept of corporality), just as his/her words, can be reported, and the place of corporal repetition in reported speech as a specific phenomenon of language. Through an examination of the semantic values of three verbs (to quote / to imitate / to ape), it argues that quoting another person's body indicates a certain degree of the subject's investment in his/her own discourse-a subject that stakes his/her social ethos on the degree of discursive success of his/her performance. 
INDEX

Mots-clés : discours rapporté, citation, analyse du discours, geste-imitation, posture

Keywords : reported speech, quotation, discourse analysis, gesture-imitation, posture

\section{AUTEUR}

\section{LAURENCE ROSIER}

Université Libre de Bruxelles, Ci-dit/Ladisco 